\title{
Vibration Characteristics of a Mistuned Bladed Disk considering the Effect of Coriolis Forces
}

\author{
Xuanen Kan and Bo Zhao \\ State Key Laboratory for Strength and Vibration of Mechanical Structures, Xian Jiaotong University, Xian 710049, China \\ Correspondence should be addressed to Xuanen Kan; kanxuanen@stu.xjtu.edu.cn
}

Received 27 March 2016; Revised 12 July 2016; Accepted 14 July 2016

Academic Editor: Jörg Wallaschek

Copyright (C) 2016 X. Kan and B. Zhao. This is an open access article distributed under the Creative Commons Attribution License, which permits unrestricted use, distribution, and reproduction in any medium, provided the original work is properly cited.

To investigate the influence of Coriolis force on vibration characteristics of mistuned bladed disk, a bladed disk with 22 blades is employed and the effects of different rotational speeds and excitation engine orders on the maximum forced response are discussed considering the effects of Coriolis forces. The results show that if there are frequency veering regions, the largest split of double natural frequencies of each modal family considering the effects of Coriolis forces appears at frequency veering region. In addition, the amplitude magnification factor considering the Coriolis effects is increased by $1.02 \%$ compared to the system without considering the Coriolis effects as the rotating speed is $3000 \mathrm{rpm}$, while the amplitude magnification factor is increased by $2.76 \%$ as the rotating speed is $10000 \mathrm{rpm}$. The results indicate that the amplitude magnification factor may be moderately enhanced with the increasing of rotating speed. Moreover, the position of the maximum forced response of bladed disk may shift from one blade to another with the increasing of the rotational speed, when the effects of Coriolis forces are considered.

\section{Introduction}

Generally, when the vibration characteristics of bladed disk are designed and analyzed, the bladed disk is assumed to be tuned [1-4]. However, practical experience shows that there are always deviations of blade-to-blade caused by manufacturing tolerances and wears during operation. These small deviations are often called mistuning of blades. Mistuning of bladed disk may cause vibration localization which may accelerate high cycle fatigue [5]. Wei and Pierre [6, 7] developed perturbation methods to investigate the vibration localization and pointed out that a small mistuning may result in strong vibration localization for weakly coupled systems. Yoo et al. [8] established a simplified pendulum model to research the influence of stiffness coupling, damping parameters on the vibration localization. Chiu and Huang [9] investigated the influence of mistuning caused by blade's stagger angle on the stability of mistuned bladed disk. Bladh et al. [10] researched the relationship between mistuning strength and amplitude magnification factor. Their results indicated that forced response amplitude and stress of mistuned bladed disk are related to mistuning strength and coupling strength.
In the previous works, the effects of Coriolis force are usually negligible. However, blades of jet engine system become thinner and geometrical shapes become more and more complicated [11]; in particular, the rotational speed becomes higher and higher. The model without considering the effects of Coriolis force cannot accurately describe the vibration characteristics of mistuned bladed disks. Therefore, Huang and Kuang [12] used Galerkin's method to research the mode localization of bladed disk considering the effects of Coriolis forces. Their results pointed out that rotating speed has a significant effect on the mode localization of mistuned bladed disk. Nikolic et al. [13] established an experiment, for the first time, to validate the split of double natural frequencies considering the effect of Coriolis forces and used a lumped parameters mass-spring model to investigate the forced response localization. The Coriolis forces of blades are related to the geometry of bladed disk. Therefore, Xin and Wang [14] used realistic bladed disk to investigate the effect of Coriolis forces on vibration characteristics and pointed out that the localization of mode shapes was significantly influenced by the Coriolis effects. 
On the other hand, if there are frequency veering regions in curves of natural frequencies versus nodal diameters, the degree of double natural frequencies split of every modal family is not thoroughly discussed in previous work. It is important for avoiding resonance of bladed disk. In addition, the effects of Coriolis forces are affected by rotational speed, but the different rotational speeds on the maximum forced response with and without considering the Coriolis effects are not thoroughly investigated in previous works.

In this paper, if there are frequency veering regions, the sensitivity of degree of double natural frequencies split of every modal family to the Coriolis effects is discussed. In addition, the effects of different rotational speeds and excitation engine orders on the maximum forced response considering the Coriolis effects are investigated.

The remaining parts of this study are organized as follows. In Section 2, the theory of rotating bladed disk with considering the effects of Coriolis force is presented. In Section 3, if there are frequency veering regions, the sensitivity of degree of double natural frequencies split of every modal family to the Coriolis effects is discussed. In Section 4, the effects of different rotational speeds and excitation engine orders on the maximum forced response considering the Coriolis effects are investigated. In Section 5, main conclusions are summarized.

\section{Theory of Rotating Blade Disk System with Considering the Coriolis Effects}

The general differential equation of motions of forced response for rotating bladed disk is described as

$$
\mathbf{M} \ddot{\mathbf{x}}(t)+(\mathbf{G}+\mathbf{C}) \dot{\mathbf{x}}(t)+\mathbf{K x}(t)=\mathbf{F}(t),
$$

where $\mathbf{M}$ is mass matrix; $\mathbf{G}$ is Coriolis matrix; $\mathbf{C}$ is damping matrix; $\mathbf{K}=\mathbf{K}_{\mathbf{o}}+\mathbf{K}_{\mathbf{C}}+\mathbf{K}_{\mathbf{m}}$, where $\mathbf{K}_{\mathbf{o}}$ is stiffness matrixes, $\mathbf{K}_{\mathbf{C}}$ is stress stiffening matrix (it is described in detail in Appendix), and $\mathbf{K}_{\mathbf{m}}$ is spin softening matrix; $\mathbf{x}(t)$ si displacement response vector of the system; $\mathbf{F}(t)$ is vector of external forces. The Coriolis matrix is generated:

$$
\mathbf{G}=2 \int_{V} \mathbf{N}^{T} \mathbf{\Omega} \mathbf{N} d V,
$$

where $\mathbf{N}$ is the shape function matrix and $\mathbf{\Omega}$ the rotational matrix:

$$
\boldsymbol{\Omega}=\left[\begin{array}{ccc}
0 & -\Omega_{z} & \Omega_{y} \\
\Omega_{z} & 0 & -\Omega_{x} \\
-\Omega_{y} & \Omega_{x} & 0
\end{array}\right] .
$$

An engine order forcing is introduced:

$$
\mathbf{F}(\theta, t)=\mathbf{F}_{0} e^{i \mathrm{EO}(\Omega t+\theta)},
$$

where $\mathbf{F}_{\mathbf{0}}$ is the force amplitude; $\mathrm{EO}$ is the engine order; $\Omega$ is the rotational speed; $t$ is the time; $\theta$ is the circumferential position.

Generally, the amplitude magnification factor is used to describe the change of forced response between mistuned and tuned bladed disk, and it is a critical parameter for evaluating high cycle fatigue of bladed disk. Quantitatively, amplitude magnification factor is the ratio between the maximum forced response of mistuned bladed disk and tuned bladed disk [15]:

$$
\text { Amplitude magnification factor }=\frac{x_{\text {maxmis }}}{x_{\text {maxtune }}},
$$

where $x_{\text {maxmis }}$ and $x_{\text {maxtune }}$ are the maximum forced response of mistuned and tuned bladed disk, respectively.

\section{Sensitivity of Degree of Double Natural Frequencies Split of Every Modal Family to the Coriolis Effects}

The effects of Coriolis forces of bladed disk are influenced by the geometrical shapes of the blade, so a realistic bladed disk of jet engine is employed in this paper. The bladed disk contains 22 blades with 330659 eight-node solid elements. The mesh of finite element of the bladed disk is shown in Figure 1. The working rotational speed is $16100 \mathrm{rpm}$. Young's modulus is $117.2 \mathrm{GPa}$, mass density is $4539.5 \mathrm{Kg} / \mathrm{m}^{3}$, Poisson's ratio is 0.3 , and the structural damping is 0.002 . Finite element method is used in the simulation.

The curves of nodal diameters versus natural frequencies of the tuned bladed disk are shown in Figure 2. It can be observed that some eigenvalues converge and then veer apart with the increasing of number of nodal diameters. This phenomenon is called frequency veering as shown in Figure 2 which is marked with dotted line. It can be seen that the first frequency veering region locates between the first and second modal family, and the second locates between the third and fourth modal family as shown in Figure 2. Mode shapes which locate in the frequency veering regions are mixed blade disk motion [16-18].

The Coriolis effects leads to the double natural frequencies split of the tuned bladed disk. Double natural frequencies split of the first modal family is shown in Figure 3(a). It can be seen that the degree of double natural frequencies split is different with the increasing of number of nodal diameters, and the largest double natural frequencies split emerges in the first order of the first nodal diameter. At the same time, the double natural frequencies split of the second modal family is shown in Figure 3(b). It shows that the largest double natural frequencies split emerges in the second order of the first nodal diameter. The first order of the first nodal diameter and the second order of the first nodal diameter locate in the first frequency veering region as shown in Figure 2.

In addition, it is interesting that the largest split of double natural frequencies of the third modal family appears at the third order of the third nodal diameter as shown in Figure 3(c). The largest split of double natural frequencies of the fourth modal family appears at the fourth order of the third nodal diameter as shown in Figure 3(d). The third order of the third nodal diameter and the fourth order of the third nodal diameter locate in the second frequency veering region as shown in Figure 2.

The results show that if there are frequency veering regions, the largest split of double natural frequencies of 


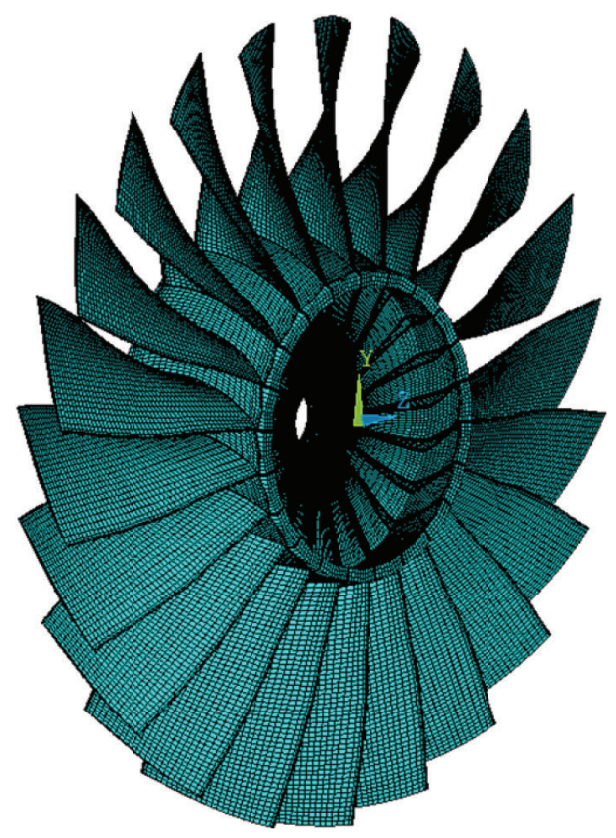

FIGURE 1: Model and mesh of finite element of the bladed disk.

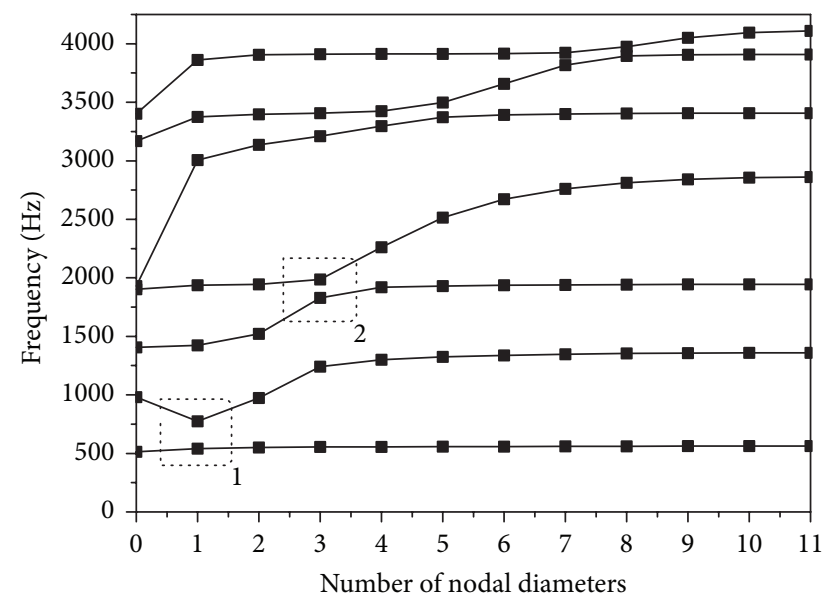

FIGURE 2: Natural frequencies versus nodal diameters.

every modal family considering the effects of Coriolis forces appears at frequency veering region. The results indicate that the effects of Coriolis force should be specially considered for avoiding resonance, if the resonance region appears nearby the frequency veering regions and it is critical for strength and vibration design of jet engine system.

The vibration displacements of bladed disk can be divided into three types as shown in Figure 4 [19]. The first type is described as disk-dominated modes in which the blade and disk have large displacements, while the blade acts like rigid plate without relevant deformations. The second type is described as coupled modes in which both the blade and disk participate in the vibration. The third type is described as blade-dominated modes in which the blade has deflect, while the disk does not participate in the vibration.
The largest split of double natural frequencies of every modal family considering the effects of Coriolis forces appears at frequency veering region, since the effects of Coriolis forces are influenced by the mode shapes. The modes in the veering regions tend to feature mixed disk-blade motion. The degree of coupling of blade and disk is higher in the veering regions. Firstly, the effects of Coriolis forces will influence the vibration of blade. Secondly, the effects of Coriolis forces influence the vibration of disk and then the vibration of disk will influence the vibration of blades. Therefore, the effects of Coriolis forces have a significant influence on vibration of modes in the veering regions. The largest split of double natural frequencies of every modal family considering the effects of Coriolis forces appears at frequency veering region.

\section{Forced Response of Mistuned Blade Disk considering the Coriolis Effects}

The harmonic analysis is applied and the full method is used in the analysis of the forced response of mistuned bladed disk considering the Coriolis effects. In this section, the influences of the Coriolis effects on the maximum forced response of mistuned bladed disk are investigated.

The first blade mode family is considered in this paper. The frequency response of tuned bladed disk under engine order 3 without considering the Coriolis effects is shown in Figure 5. We can see that the frequency response of blade is identical with each other and there is only one peak. It is because that tuned bladed disk is cyclic symmetric structure, and energy can uniformly transmit between each blade. Hence the frequency response of blade is identical with each other. Moreover, the engine order 3 will excite the vibration with the third nodal diameter, in the tuned bladed disk [20]. Therefore, the frequency response curve has only one peak. 


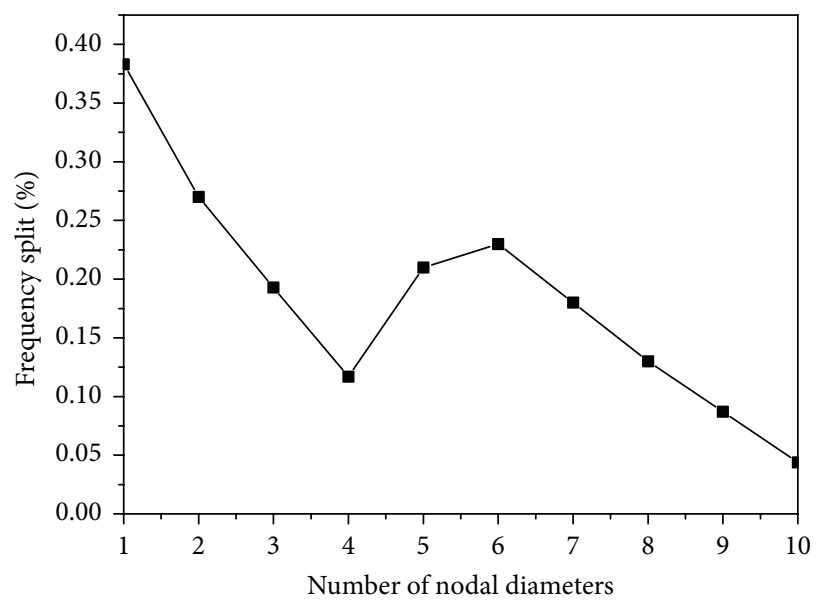

(a) The first modal family

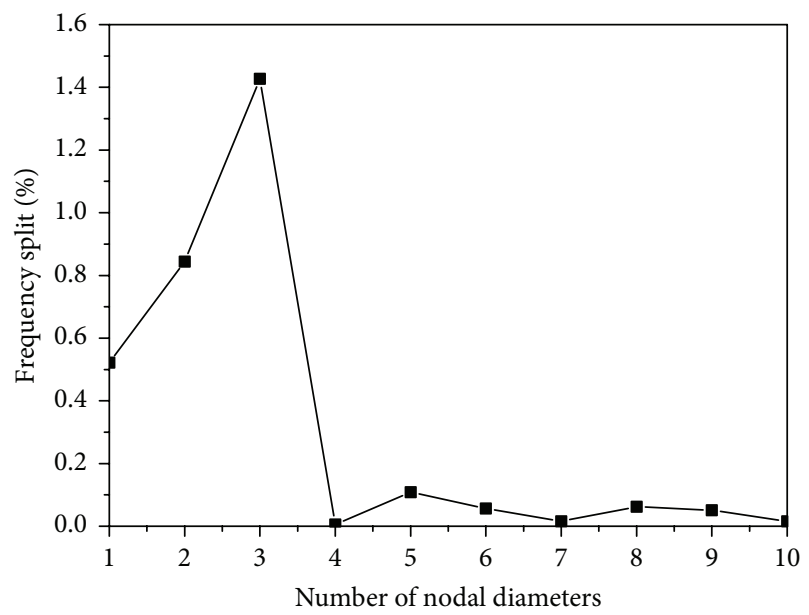

(c) The third modal family

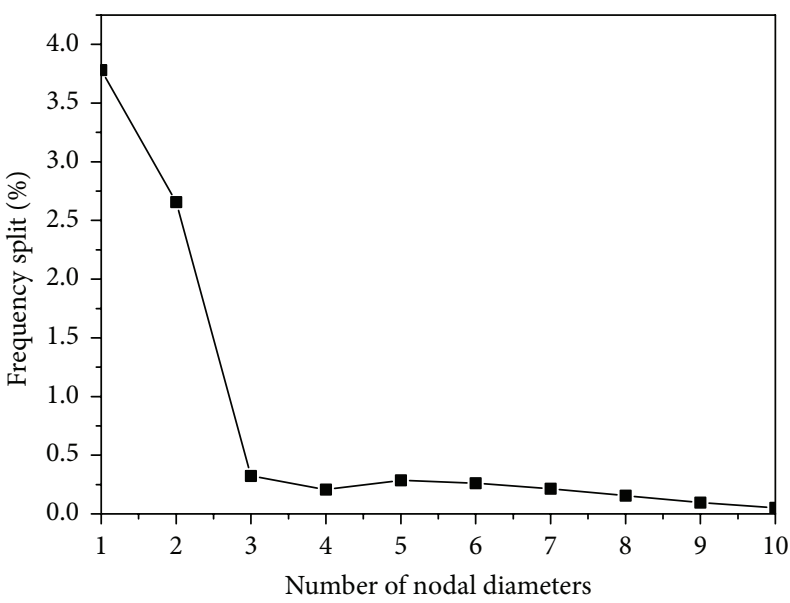

(b) The second modal family

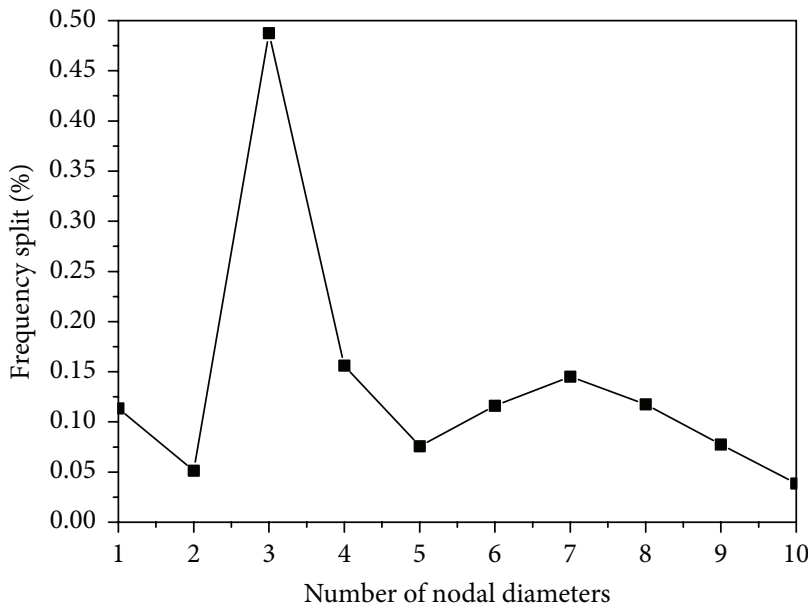

(d) The fourth modal family

FIGURE 3: Double natural frequencies split.

There is always mistuning of blade due to manufacturing tolerances and wear in operation. Mistuning of blades will break the cyclic symmetric properties and will lead to the split of double natural frequencies of tuned bladed disk. The effect of Coriolis forces can also lead to the split of double natural frequencies of tuned bladed disk. So, it is interesting to know the change of the maximum forced response of mistuned bladed disk with and without considering the Coriolis effects.

Mistuning of the blades is introduced by allowing each blade to have different Young's modulus. In the simulation, Young's modulus of the blades satisfies the following relationship:

$$
E_{j}=\left(1+\delta_{j}\right) E_{0}, \quad j=1, \ldots, 22,
$$

where $E_{0}$ and $E_{j}$ are Young's modulus for the $j$ th blade of tuned and mistuned bladed disk. $\delta_{j}$ is the mistuning ratio and the dimensionless mistuning parameter. $\delta_{j}$ is randomly obtained from a normal distribution with standard deviation of 1 percent and mean value 0 as shown in Figure 6.

The frequency response of the mistuned bladed disk without considering the Coriolis effects under EO 3 forcing is shown in Figure 7. It can be observed that each blade has a different frequency response curve and multipeaks emerging. Mistuning of blade destroys the cyclic symmetric properties of blade disk system and the energy cannot uniformly transmit. Energy is confined to a few blades which will lead to vibration localization of bladed disk.

The frequency response of mistuned bladed disk considering the effect of Coriolis forces under EO 3 forcing is shown in Figure 8. It can be seen that each blade has a different frequency response curve and multipeaks emerging.

The amplitude magnification factor of mistuned bladed disk considering the Coriolis effects is increased by $2.13 \%$ compared to the system without considering the Coriolis effects. At the same time, the maximum forced response of each blade will vary when the Coriolis effects is considered as shown in Figure 9. The maximum variation of forced response appears at the 6 th blade and it is increased by $34.6 \%$ when the Coriolis effects are considered.

4.1. Effects of Excitation Engine Order on the Maximum Forced Response considering the Coriolis Effects. The vector of external forces are related to engine order, so in this section, 


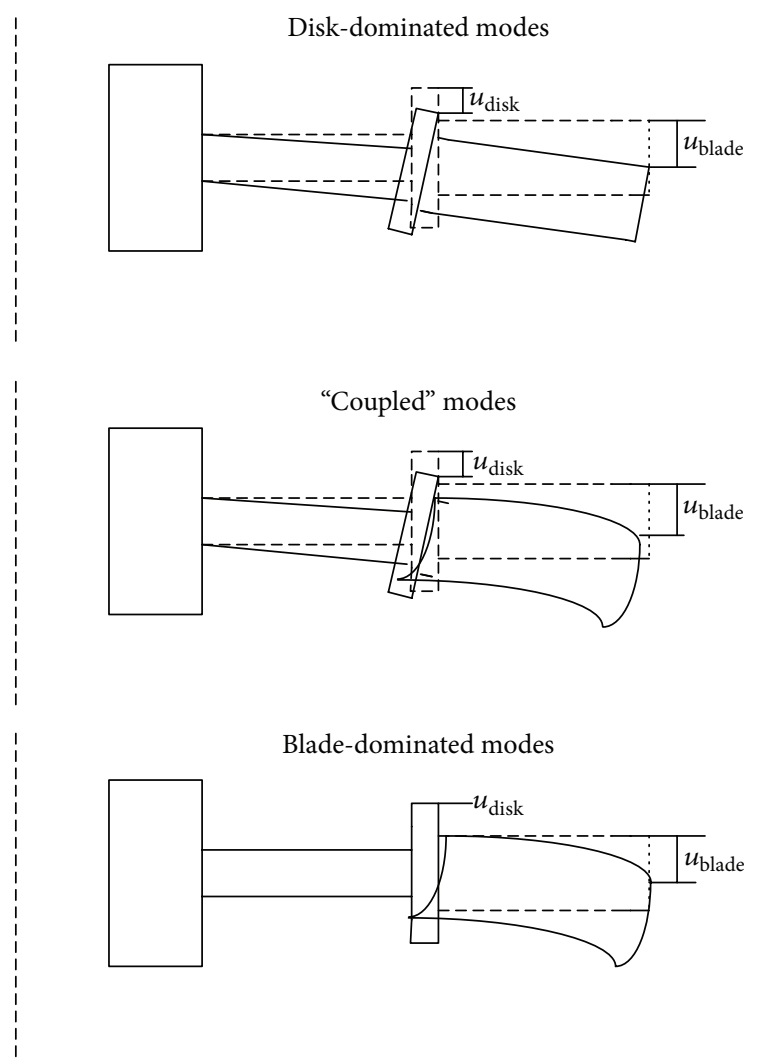

Figure 4: Blade and disk deflection for different vibration mode.

TABLE 1: The maximum forced response of mistuned bladed disk with and without considering the Coriolis effects.

\begin{tabular}{lccc}
\hline Engine order & $A_{N C}$ & $A_{C}$ & $\left|\left(A_{C}-A_{N C}\right) / A_{N C}\right|(\%)$ \\
\hline 1 & 2.9209 & 2.7608 & 5.481 \\
-1 & 3.0343 & 2.6367 & 13.104 \\
3 & 2.7349 & 2.7932 & 2.1317 \\
-3 & 2.6121 & 2.6739 & 2.3659 \\
11 & 2.5679 & 2.6927 & 4.86 \\
-11 & 2.5679 & 2.6927 & 4.86 \\
\hline
\end{tabular}

the effects of engine order on the maximum forced response are discussed with considering the Coriolis effects.

The maximum forced response with and without considering the Coriolis effects under different excitation engine orders is shown in Table 1. $A_{N C}$ is the maximum forced response without considering the Coriolis effects, and $A_{C}$ is the maximum forced response with considering the Coriolis effects. It can be observed that the maximum forced response of EO 11 is identical with EO -11, while the maximum forced responses of mistuned bladed disk excited by other engine orders are different.

(1) The natural frequencies are double natural frequencies of the tuned bladed disk except for the 0 and $N / 2$ ( $N$ is even) nodal diameters. There are 22 blades in our model of bladed disk system. Hence, the mode shapes at nodal diameter 11 are single mode. Moreover, the back traveling excitation by EO 11 is coincident with the forward traveling excitation by EO -11 .

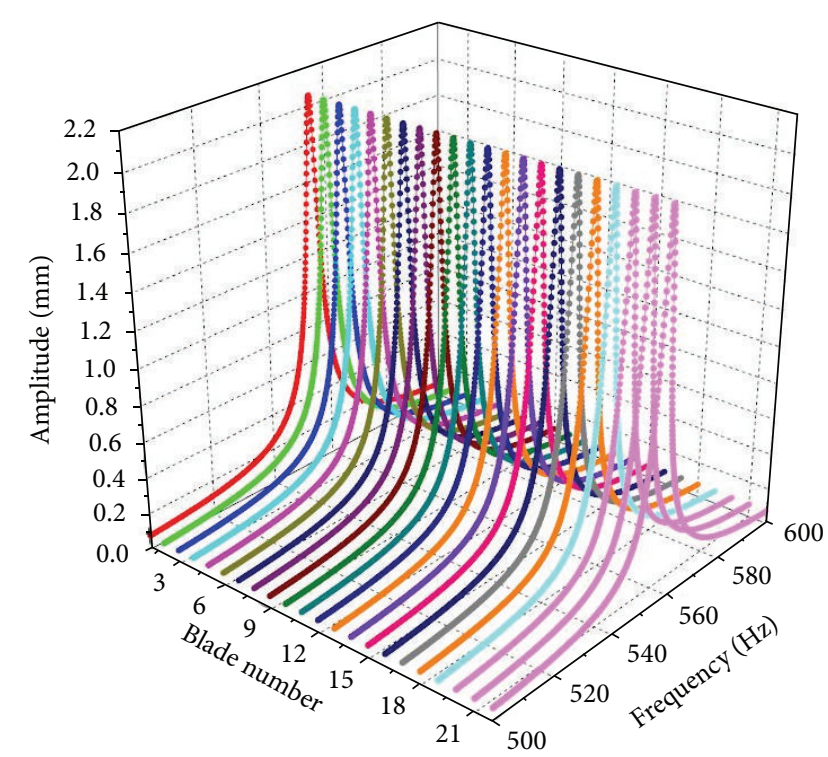

FIGURE 5: Frequency response of tuned bladed disk without considering the Coriolis effects under EO 3.

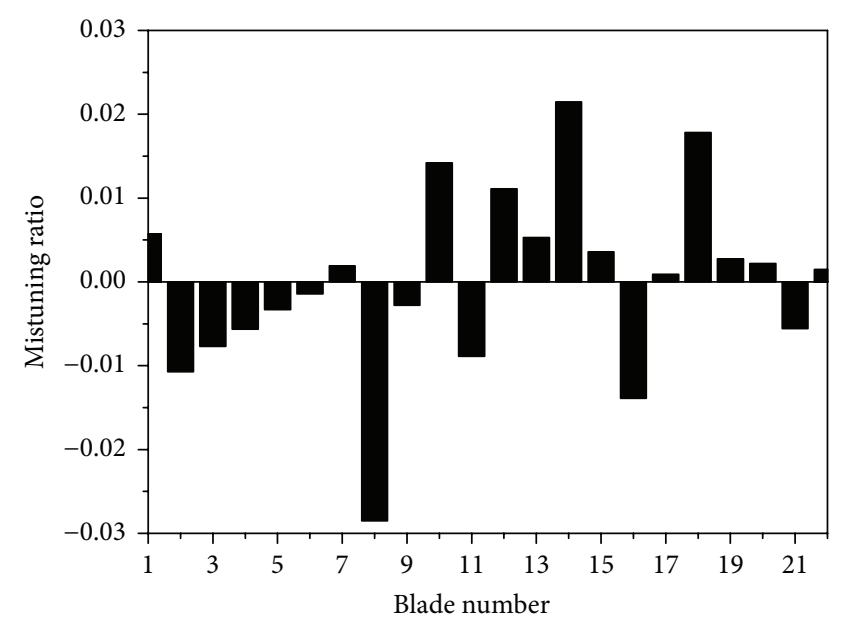

FIGURE 6: Mistuning pattern.

The forced response curve of the mistuned bladed disk excited by EO 11 is identical with the forced response curve excited by EO - 11. Therefore, the maximum forced response is identical with each other excited by EO 11 and EO -11 .

(2) The mode shapes at 1 to 10 nodal diameters are double modes. The effect of Coriolis force will lead to split of the double natural frequencies. Moreover, the back traveling excitation EO 1 and EO 3 are different with the forward traveling excitation EO -1 and EO -3 . Therefore, frequency response curves excited by EO 1 and EO 3 are different with the frequency response curves excited by $\mathrm{EO}-1$ and $\mathrm{EO}-3$, respectively.

\subsection{Effects of Different Rotational Speeds on the Maximum} Forced Response with and without Considering the Coriolis Effects. The Coriolis forces are affected by the different rotational speeds. In this section, the effects of different rotational 


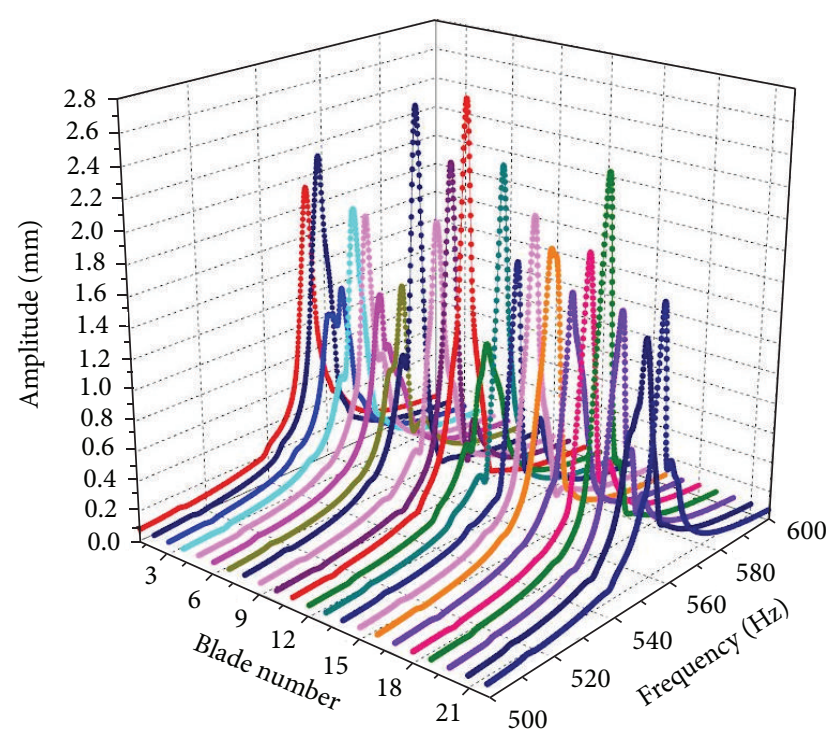

Figure 7: The frequency response of mistuned bladed disk without considering the Coriolis effects under EO 3.

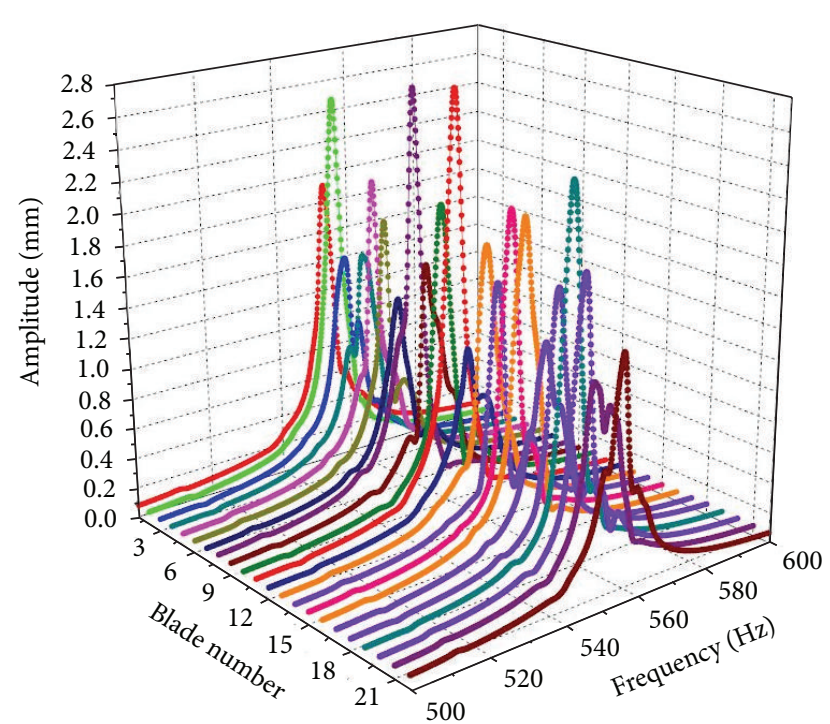

FIGURE 8: The frequency response of mistuned bladed disk considering the Coriolis effects under EO 3.

speeds on the maximum forced response are investigated considering the Coriolis effects.

The forced response amplitude magnification factor with and without the effect of Coriolis forces under different rotational speeds is shown in Figure 10. The amplitude magnification factor considering the Coriolis effects is increased by $1.02 \%$ compared to the system without considering the effect of Coriolis forces as the rotating speed is $3000 \mathrm{rpm}$. The amplitude magnification factor considering the effect of Coriolis forces is increased by $2.76 \%$ compared to the system without considering the Coriolis effects as the rotating speed is $10000 \mathrm{rpm}$.

At the same time, the maximum forced response of each blade varies, when the effect of Coriolis force is considered.

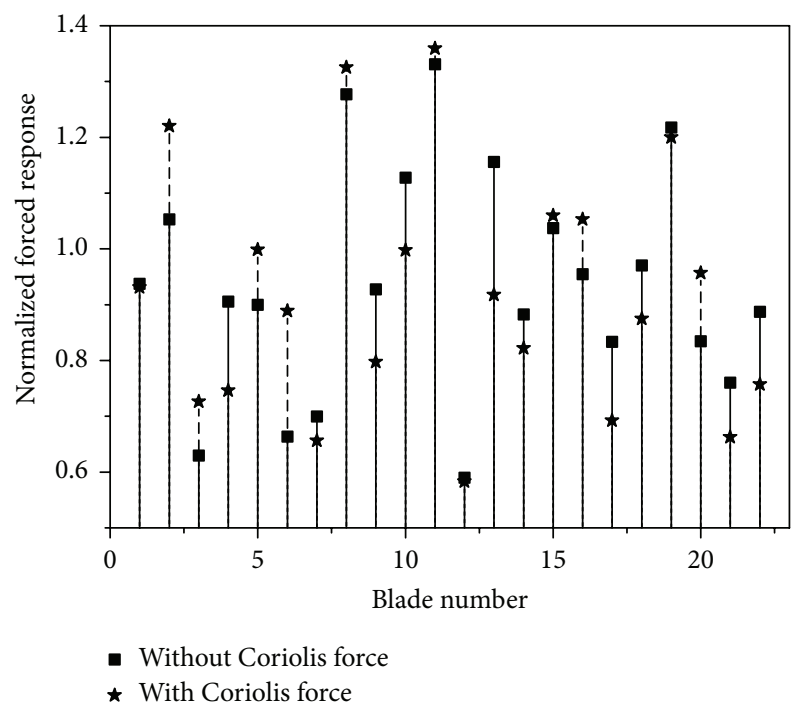

Figure 9: The maximum forced response of each blade with and without considering the Coriolis effects.

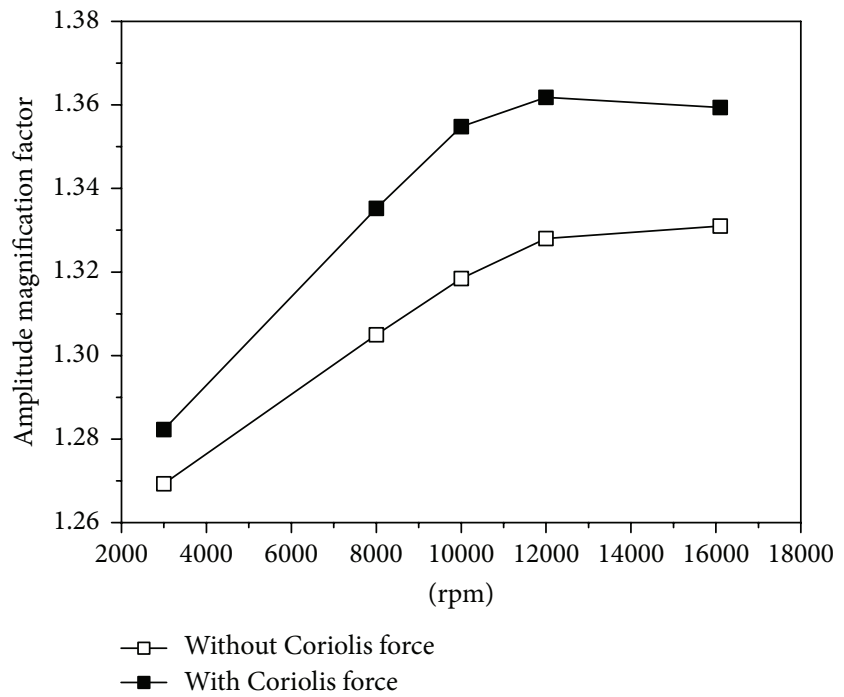

FIGURE 10: The amplitude magnification factor with and without considering the effect of Coriolis forces under different rotational speeds.

The largest change of the maximum forced response considering the effect of Coriolis forces appears at the 6th blade, and the maximum forced response of the 6th blade is increased by $6.56 \%$ compared to the system without considering the effect of Coriolis forces when the rotational speed is $3000 \mathrm{rpm}$ as shown in Figure 11(a). The largest change of maximum forced response considering the effect of Coriolis forces appears at the 6th blade, and the maximum forced response of the 6th blade is increased by $25.5 \%$ compared to the system without considering the effect of Coriolis forces when the rotational speed is $10000 \mathrm{rpm}$ as shown in Figure 11(b).

Another interesting phenomenon found that the position of the maximum forced response of the mistuned bladed disk shifts from the 11th blade to the 8th blade with the effects 


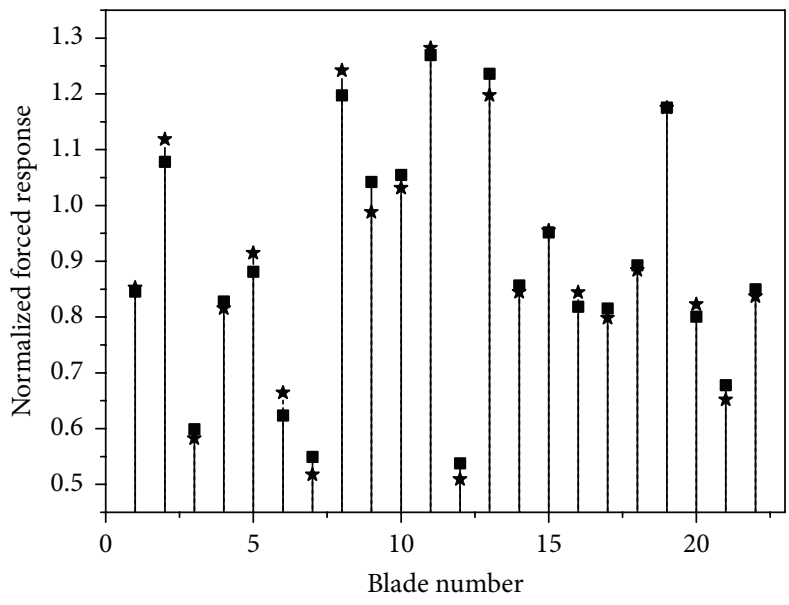

- Without Coriolis force

$\star$ With Coriolis force

(a) Rotating speed is $3000 \mathrm{rpm}$

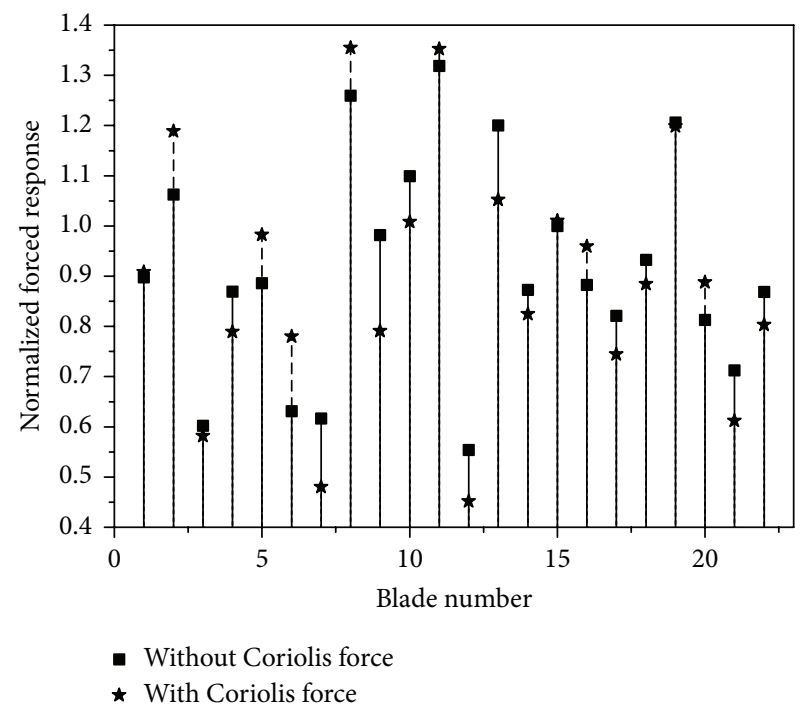

(b) Rotating speed is $10000 \mathrm{rpm}$

Figure 11: The maximum forced response of each blade with and without considering the Coriolis effects.

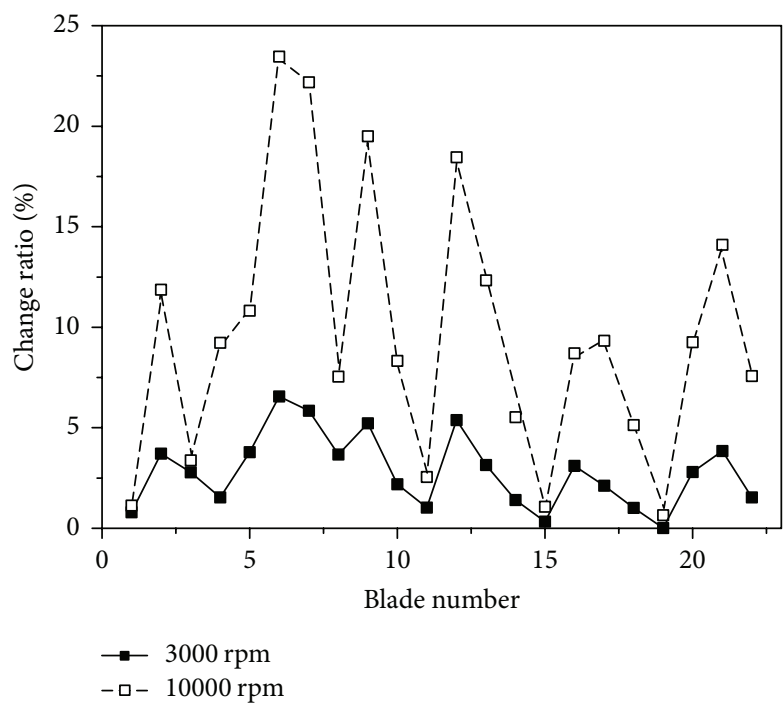

FIGURE 12: The average change ratio of amplitude of each blade when the rotating speed is $3000 \mathrm{rpm}$ and $10000 \mathrm{rpm}$.

of Coriolis forces being considered, when the rotating speed is $10000 \mathrm{rpm}$, as shown in Figure 11(b). The average change ratio of amplitude of each blade with the rotational speed $3000 \mathrm{rpm}$ and $10000 \mathrm{rpm}$ is shown in Figure 12. In order to quantitatively describe the change of each blade with and without considering the Coriolis effects, a new parameter is introduced:

$$
P_{r}=\frac{\sum_{i=1}^{N}\left|\left(A_{r i}^{C}-A_{r i}^{N C}\right) / A_{r i}^{N C}\right|}{N},
$$

where $P_{r}$ is the average change of amplitude of each blade when the rotating speed is $r \mathrm{rpm}, A_{r i}^{\mathrm{C}}$ and $A_{r i}^{N C}$ are the amplitude of $i$ th blade with and without considering the Coriolis effects, and $N$ is the total number of blades. When the rotating speed is $3000 \mathrm{rpm}$ and $10000 \mathrm{rpm}, P_{r}$ is $2.77 \%$ and $9.64 \%$, respectively.

Previous work [12] indicated that rotating speed has a significant effect on mode localization of mistuned bladed disk. The results of the present paper give some new conclusions as follows:

(1) The maximum forced response of bladed disk may be enhanced with the increasing of rotating speed.

(2) The position of the maximum of forced response considering the Coriolis effects may shift from one blade to another with the increasing of rotational speed.

(3) A new parameter is introduced to quantitatively describe the average change of amplitude of each blade with and without considering the Coriolis effects.

\section{Conclusions}

The influences of Coriolis effects on vibration characteristics of a mistuned bladed disk have been investigated and the major conclusions of this paper are as follows:

(1) If there are frequency veering regions in curves of natural frequencies versus nodal diameters, the largest double natural frequencies split of every modal family appears at frequency veering regions. The results indicate that the effect of Coriolis force should be specially considered for avoiding resonance of bladed disk, if the resonance region appears nearby the frequency veering regions.

(2) The maximum forced response of bladed disk and the maximum forced response of each blade will vary when the effects of Coriolis force are considered 


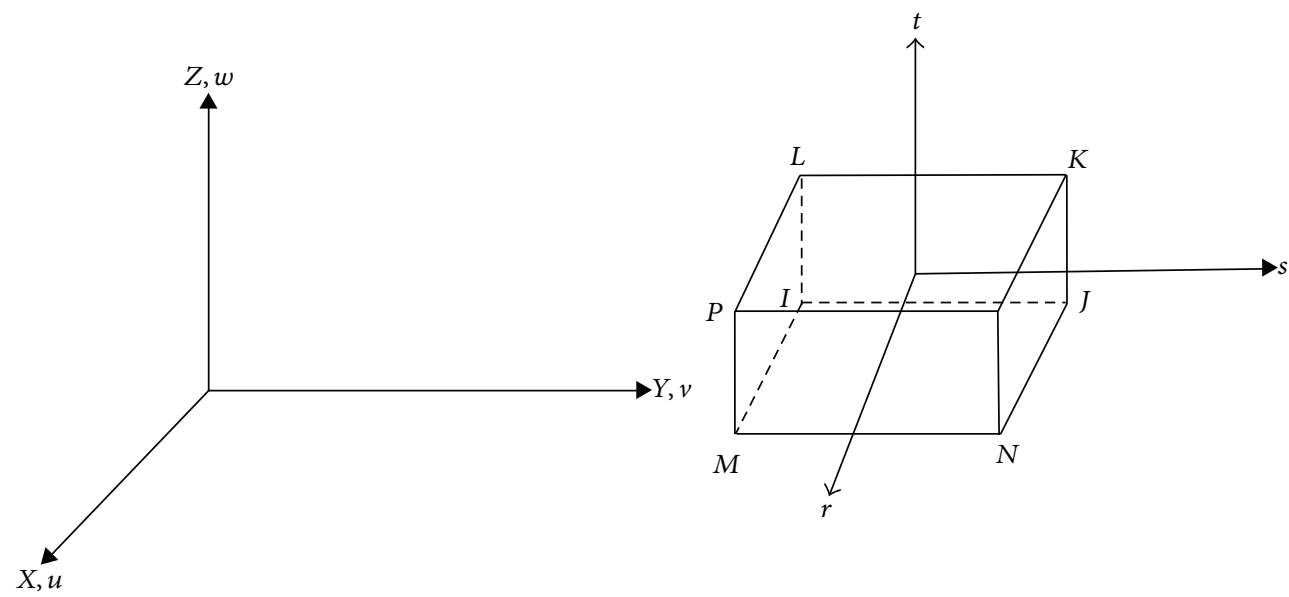

FIGURE 13: Eight-node brick solid element.

and this variation is related to the excitation engine orders. Furthermore, the position of the maximum forced response of bladed disk will shift from one blade to another, as the effects of Coriolis forces are considered.

(3) The amplitude magnification factor considering the effect of Coriolis forces is increased by $1.02 \%$ compared to the system without considering the effects of Coriolis forces as the rotating speed is $3000 \mathrm{rpm}$, while the amplitude magnification factor is increased by $2.76 \%$ as the rotating speed is $10000 \mathrm{rpm}$. The results indicate that the maximum forced response may be moderately enhanced with the increasing of rotational speed. Furthermore, the position of the maximum forced response considering the effects of Coriolis forces may shift with the increasing of rotational speed.

(4) A new parameter is introduced to quantitatively describe the average change of amplitude of each blade with and without considering the Coriolis effects.

\section{Appendix}

In this paper, $\mathbf{K}_{\mathbf{C}}$ is the stress stiffening matrix. $N$ is the shape function of 8-node brick solid element as shown in Figure 13:

$$
\mathbf{K}_{\mathbf{C}}=\left[\begin{array}{lll}
\mathbf{S}_{0} & & \\
& \mathbf{S}_{0} & \\
& & \mathbf{S}_{0}
\end{array}\right] \text {, }
$$

where

$$
\begin{gathered}
\mathbf{S}_{0}=\int_{v} \mathbf{S}_{\mathrm{g}}{ }^{\mathrm{T}} \mathbf{S}_{\mathrm{m}} \mathbf{S}_{\mathrm{g}} d v, \\
\mathbf{S}_{\mathrm{m}}=\left[\begin{array}{ccc}
\sigma_{\mathrm{x}} & \sigma_{\mathrm{xy}} & \sigma_{\mathrm{xz}} \\
\sigma_{\mathrm{xy}} & \sigma_{\mathrm{y}} & \sigma_{\mathrm{yz}} \\
\sigma_{\mathrm{xz}} & \sigma_{\mathrm{yz}} & \sigma_{\mathrm{z}}
\end{array}\right],
\end{gathered}
$$

$$
\begin{aligned}
& \mathbf{S}_{\mathbf{g}}=\left[\begin{array}{llll}
\frac{\partial N_{1}}{\partial x} & \frac{\partial N_{2}}{\partial x} & \cdots & \frac{\partial N_{8}}{\partial x} \\
\frac{\partial N_{1}}{\partial y} & \frac{\partial N_{2}}{\partial y} & \cdots & \frac{\partial N_{8}}{\partial y} \\
\frac{\partial N_{1}}{\partial \mathbf{z}} & \frac{\partial N_{2}}{\partial \mathbf{z}} & \cdots & \frac{\partial N_{8}}{\partial \mathbf{z}}
\end{array}\right], \\
& N=\left[\begin{array}{c}
u \\
v \\
w
\end{array}\right],
\end{aligned}
$$

where

$$
\begin{aligned}
u= & \frac{1}{8}\left(u_{I}(1-s)(1-t)(1-r)\right. \\
& +u_{J}(1+s)(1-t)(1-r) \\
& +u_{K}(1+s)(1+t)(1-r) \\
& +u_{L}(1-s)(1+t)(1-r) \\
& +u_{M}(1-s)(1-t)(1+r) \\
& +u_{N}(1+s)(1-t)(1+r) \\
& +u_{O}(1+s)(1+t)(1+r) \\
& \left.+u_{P}(1-s)(1+t)(1+r)\right), \\
v & \frac{1}{8}\left(v_{I}(1-s)(1-t)(1-r)\right. \\
& +v_{J}(1+s)(1-t)(1-r) \\
& +v_{K}(1+s)(1+t)(1-r) \\
& +v_{L}(1-s)(1+t)(1-r) \\
& +v_{M}(1-s)(1-t)(1+r) \\
& +v_{N}(1+s)(1-t)(1+r)
\end{aligned}
$$




$$
\begin{aligned}
& +v_{O}(1+s)(1+t)(1+r) \\
& \left.+v_{P}(1-s)(1+t)(1+r)\right) \\
w & =\frac{1}{8}\left(w_{I}(1-s)(1-t)(1-r)\right. \\
& +w_{J}(1+s)(1-t)(1-r) \\
& +w_{K}(1+s)(1+t)(1-r) \\
& +w_{L}(1-s)(1+t)(1-r) \\
& +w_{M}(1-s)(1-t)(1+r) \\
& +w_{N}(1+s)(1-t)(1+r) \\
& +w_{O}(1+s)(1+t)(1+r) \\
& \left.+w_{P}(1-s)(1+t)(1+r)\right) .
\end{aligned}
$$

\section{Competing Interests}

The authors declare that they have no competing interests.

\section{References}

[1] I. Y. Shen, "Vibration of rotationally periodic structures," Journal of Sound and Vibration, vol. 172, no. 4, pp. 459-470, 1994.

[2] J. Tang and K. W. Wang, "Vibration control of rotationally periodic structures using passive piezoelectric shunt networks and active compensation," Journal of Vibration and Acoustics, Transactions of the ASME, vol. 121, no. 3, pp. 379-390, 1999.

[3] J. Y. Chang and J. A. Wickert, "Response of modulated doublet modes to travelling wave excitation," Journal of Sound and Vibration, vol. 242, no. 1, pp. 69-83, 2001.

[4] J. Y. Chang and J. A. Wickert, "Measurement and analysis of modulated doublet mode response in mock bladed disks," Journal of Sound and Vibration, vol. 250, no. 3, pp. 379-400, 2002.

[5] M. P. Castanier and C. Pierre, "Modeling and analysis of mistuned bladed disk vibration: current status and emerging directions," Journal of Propulsion and Power, vol. 22, no. 2, pp. 384-396, 2006.

[6] S.-T. Wei and C. Pierre, "Localization phenomena in mistuned assemblies with cyclic symmetry-part I: free vibrations," Journal of Vibration, Acoustics, Stress, and Reliability in Design, vol. 110, no. 4, pp. 429-438, 1988.

[7] S.-T. Wei and C. Pierre, "Localization phenomena in mistuned assemblies with cyclic symmetry. Part II: forced vibrations," Journal of Vibration, Acoustics, Stress, and Reliability in Design, vol. 110, no. 4, pp. 439-449, 1988.

[8] H. H. Yoo, J. Y. Kim, and D. J. Inman, "Vibration localization of simplified mistuned cyclic structures undertaking external harmonic force," Journal of Sound and Vibration, vol. 261, no. 5, pp. 859-870, 2003.

[9] Y.-J. Chiu and S.-C. Huang, "The influence of a mistuned blade's staggle angle on the vibration and stability of a shaft-disk-blade assembly," Shock and Vibration, vol. 15, no. 1, pp. 3-17, 2008.

[10] R. Bladh, C. Pierre, M. P. Castanier, and M. J. Kruse, "Dynamic response predictions for a mistuned industrial turbomachinery rotor using reduced-order modeling," Journal of Engineering for Gas Turbines and Power, vol. 124, no. 2, pp. 311-324, 2002.

[11] C. Gibert, V. Kharyton, F. Thouverez, and P. Jean, "On forced response of a rotating integrally bladed disk: predictions and experiments," in Proceedings of the ASME Turbo Expo Power for Land, Sea, and Air, pp. 1103-1116, Glasgow, UK, June 2010.

[12] B. W. Huang and J. H. Kuang, "Mode localization in a rotating mistuned turbo disk with Coriolis effect," International Journal of Mechanical Sciences, vol. 43, no. 7, pp. 1643-1660, 2001.

[13] M. Nikolic, E. P. Petrov, and D. J. Ewins, "Coriolis forces in forced response analysis of mistuned bladed disks," Journal of Turbomachinery, vol. 129, no. 4, pp. 730-739, 2007.

[14] J. Xin and J. Wang, "Investigation of coriolis effect on vibration characteristics of a realistic mistuned bladed disk," in Proceedings of the ASME Turbo Expo: Turbine Technical Conference and Exposition, pp. 993-1005, Vancouver, Canada, June 2011.

[15] M. P. Castanier and C. Pierre, "Investigation of the combined effects of intentional and random mistuning on the forced response of bladed disks," in Proceedings of the 34th AIAA/ASME/SAE/ASEE Joint Propulsion Conference and Exhibit, vol. 1001, Cleveland, Ohio, USA, 1998.

[16] J. L. Du Bois, S. Adhikari, and N. A. J. Lieven, "On the quantification of eigenvalue curve veering: a veering index," Journal of Applied Mechanics, vol. 78, no. 4, Article ID 041007, 2011.

[17] J. A. Kenyon, J. H. Griffin, and N. E. Kim, "Sensitivity of tuned bladed disk response to frequency veering," Journal of Engineering for Gas Turbines and Power, vol. 127, no. 4, pp. 835$842,2005$.

[18] I. Lopez, R. R. J. J. van Doorn, R. van der Steen, N. B. Roozen, and $\mathrm{H}$. Nijmeijer, "Frequency loci veering due to deformation in rotating tyres," Journal of Sound and Vibration, vol. 324, no. 3-5, pp. 622-639, 2009.

[19] T. Klauke, U. Strehlau, and A. Kühhorn, "Integer frequency veering of mistuned blade integrated disks," Journal of Turbomachinery, vol. 135, no. 3, Article ID 061004, 2013.

[20] S. J. Wildheim, "Excitation of rotationally periodic structures," Journal of Applied Mechanics, vol. 46, no. 4, pp. 878-882, 1979. 


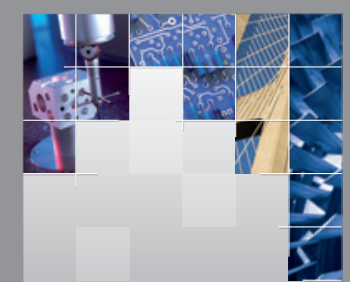

\section{Enfincering}
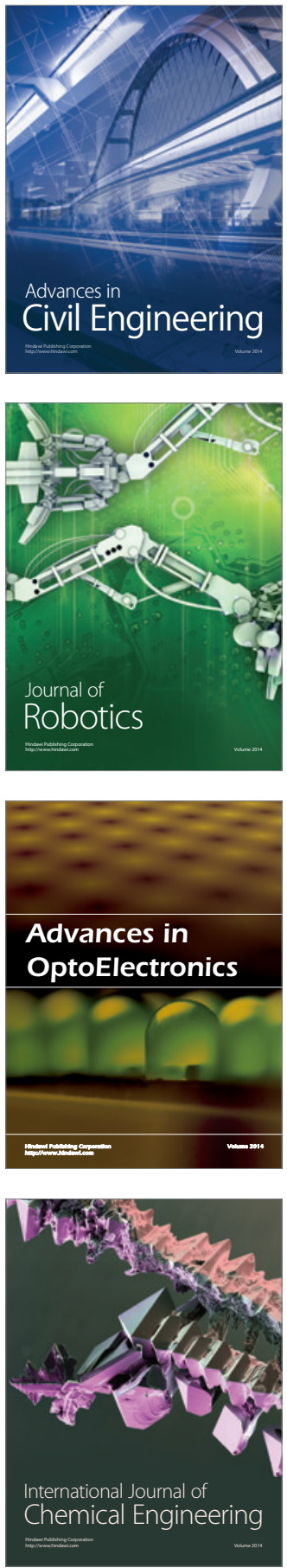

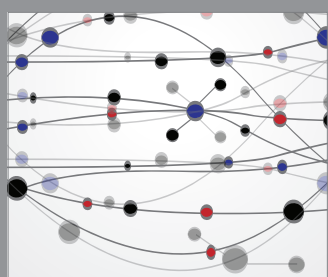

The Scientific World Journal

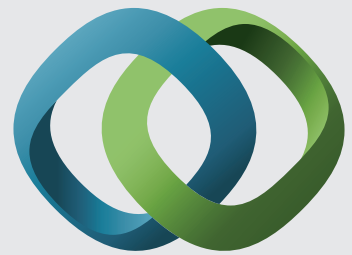

\section{Hindawi}

Submit your manuscripts at

http://www.hindawi.com
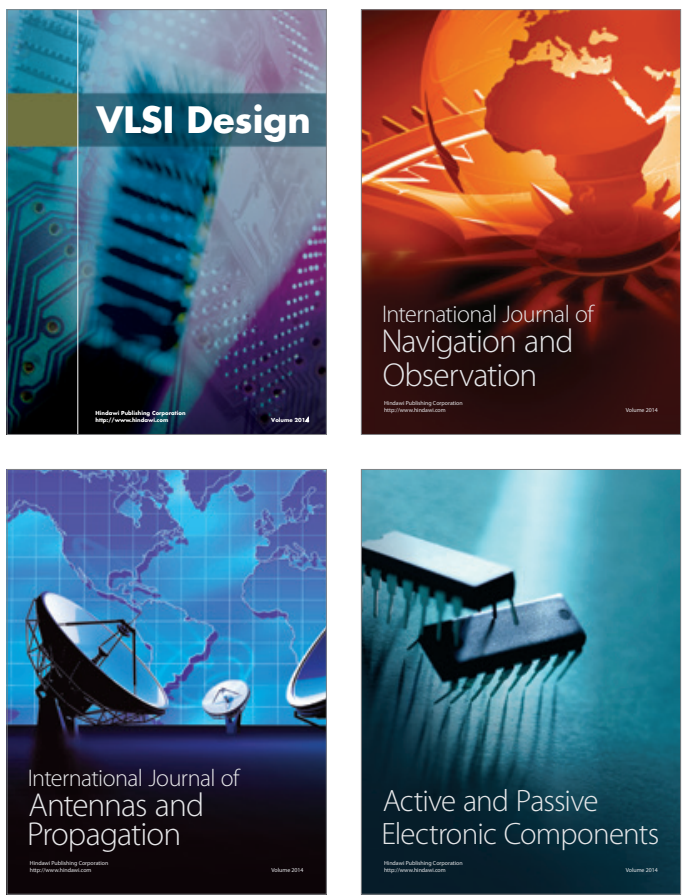
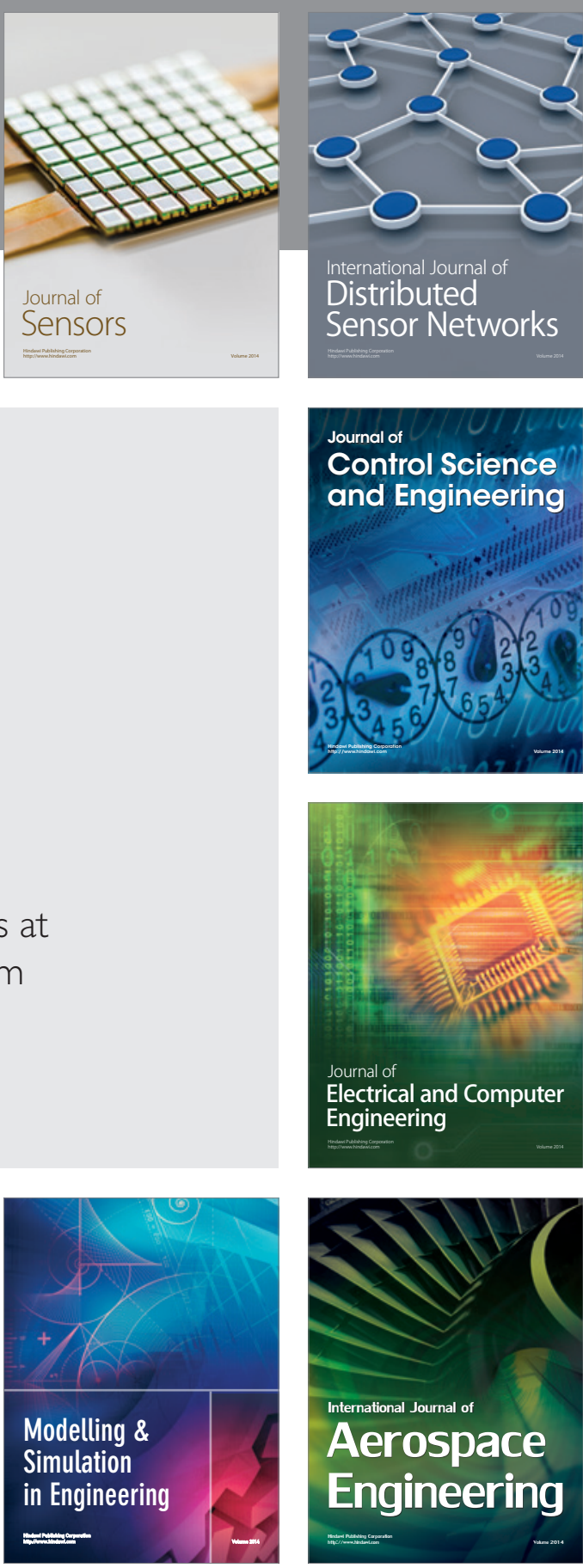

International Journal of

Distributed

Sensor Networks

Journal of

Control Science

and Engineering
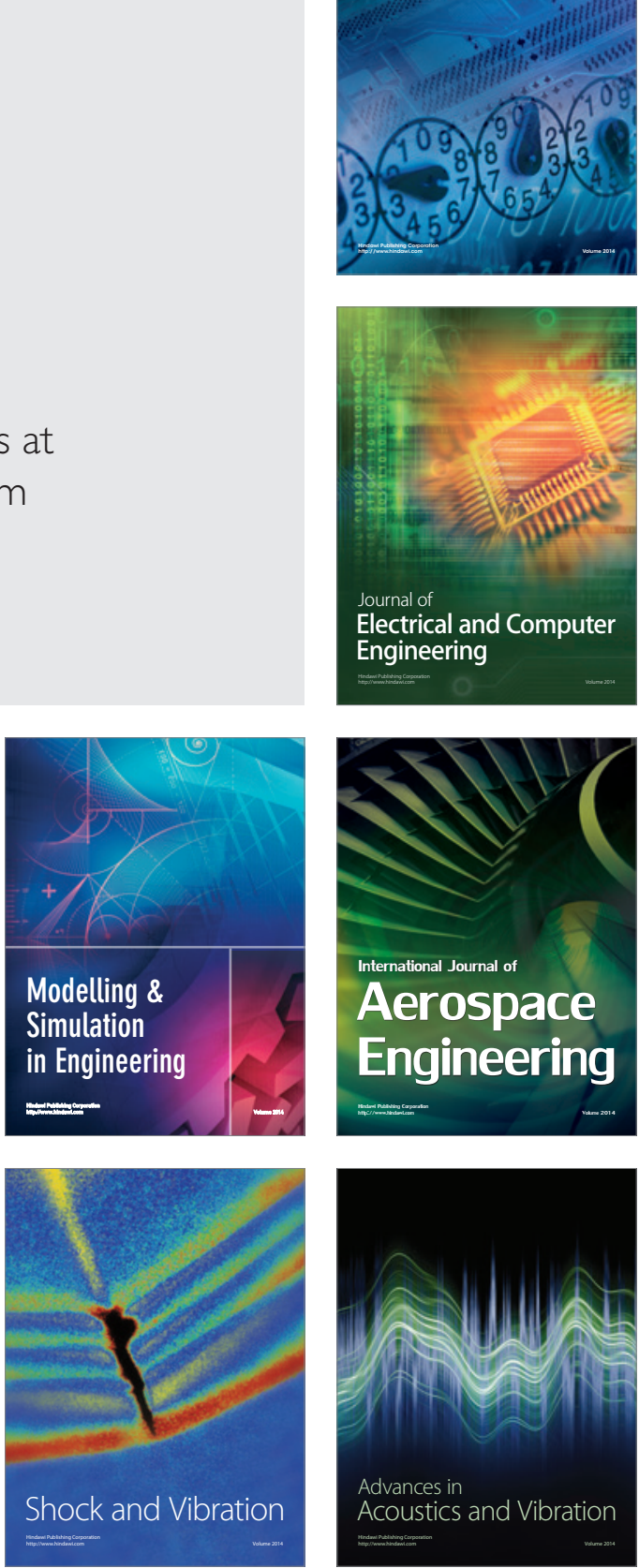